\title{
Fermi-LAT Observations of $\gamma$-Ray Emission Towards the Outer Halo of M31
}

\author{
Chris Karwin*, Simona Murgia, and Sheldon Campbell \\ Department of Physics and Astronomy, University of California, Irvine, CA 92697, USA \\ E-mail: ckarwinduci.edu, smurgiaduci.edu, sheldoceuci.edu

\section{Igor Moskalenko} \\ Hansen Experimental Physics Laboratory and Kavli Institute for Particle Astrophysics and \\ Cosmology, Stanford University, Stanford, CA 94305, USA \\ E-mail: imosestanford.edu
}

\begin{abstract}
The Andromeda galaxy is the closest spiral galaxy to us and has been the subject of numerous studies. It harbors a massive dark matter halo which may span up to $\sim 600 \mathrm{kpc}$ across and comprises $\sim 90 \%$ of the galaxy's total mass. This halo size translates into a large diameter of $42^{\circ}$ on the sky for an M31-Milky Way (MW) distance of $785 \mathrm{kpc}$, but its presumably low surface brightness makes it challenging to detect with $\gamma$-ray telescopes. Using 7.6 years of Fermi Large Area Telescope (Fermi-LAT) observations, we make a detailed study of the $\gamma$-ray emission between $1-$ $100 \mathrm{GeV}$ towards M31's outer halo, with a total field radius of $60^{\circ}$ centered at M31, and perform an in-depth analysis of the systematic uncertainties related to the observations. We use the cosmic ray propagation code GALPROP to construct specialized interstellar emission models to characterize the foreground $\gamma$-ray emission from the MW, including a self-consistent determination of the isotropic component. We find evidence for an extended excess that appears to be distinct from the conventional MW foreground, having a total radial extension upwards of $\sim 120-200 \mathrm{kpc}$ from the center of M31. We discuss plausible interpretations of the excess emission but emphasize that uncertainties in the MW foreground, and in particular, modeling of the H I-related components, have not been fully explored and may impact the results.
\end{abstract}

36th International Cosmic Ray Conference -ICRC2019-

July 24th - August 1st, 2019

Madison, WI, U.S.A.

${ }^{*}$ Speaker. 


\section{Introduction}

The goal of this work is to search for extended $\gamma$-ray emission originating beyond the galactic disk of M31, and to examine the implications for cosmic rays (CRs) and dark matter (DM). There are two primary motivations for this search. First, CR interactions with the radiation field of M31's stellar halo and/or the circumgalactic gas could generate a detectable signal in $\gamma$-rays. Second, M31's DM halo has a large extension on the sky and could produce a detectable signal within currently allowed DM scenarios, which would be complementary to other targets-and specifically, the Galactic center. What makes M31 advantageous in regards to DM searches with $\gamma$-rays is that the entire DM halo is seen from the outside, so we see the extended integral signal. For the MW we see through the halo, and so it can be easily confused with diffuse components. Our primary field of interest is a $28^{\circ} \times 28^{\circ}$ square region, which amounts to a projected radius of $\sim 200 \mathrm{kpc}$ from the center of M31. Our study complements previously published results on M31 [1-4] and is the first to explore the farthest reaches of the M31 system in $\gamma$-rays. The full analysis is presented in [5].

\section{Observations}

Our full region of interest (ROI) corresponds to a radius of $60^{\circ}$ centered at the position of $\mathrm{M} 31,(l, b)=\left(121.17^{\circ},-21.57^{\circ}\right)$. We employ front and back converting events corresponding to the P8R2_CLEAN_V6 selection. The events have energies in the range 1-100 GeV and have been collected from 2008-08-04 to 2016-03-16 (7.6 years). The data are divided into 20 bins equally spaced in logarithmic energy, with $0.2^{\circ} \times 0.2^{\circ}$ pixel size. The analysis is carried out with the Fermi-LAT ScienceTools (version v10r0p5) ${ }^{1}$. In particular, the binned maximum likelihood fits are performed with the gtlike package.

Figure 1 shows the total observed counts between 1-100 GeV for the full ROI. Two different count ranges are displayed. The map on the left shows the full range. The bright emission along $0^{\circ}$ latitude corresponds to the plane of the MW. The map on the right shows the saturated counts map, emphasizing the lower counts at higher latitudes. Overlaid is a green dashed circle $\left(21^{\circ}\right.$ in radius) corresponding to a $300 \mathrm{kpc}$ projected radius centered at M31, for an M31-MW distance of $785 \mathrm{kpc}$, i.e. the canonical virial radius of M31. Also shown is M31's population of dwarf galaxies. The primary purpose of the overlay is to provide a qualitative representation of the extent of M31's outer halo, and to show its relationship to the MW disk. Note that we divide the full ROI into subregions, and our primary field of interest is a $28^{\circ} \times 28^{\circ}$ square region centered at M31, which we refer to as field M31 (FM31).

\section{Interstellar Emission Models}

The foreground emission from the MW and the isotropic component (the latter includes unresolved extragalactic diffuse $\gamma$-ray emission, residual instrumental background, and possibly contributions from other Galactic components which have a roughly isotropic distribution) are the dominant contributions in $\gamma$-rays towards the M31 region. We use the CR propagation code GAL$\mathrm{PROP}^{2}$ (v56) [6,7] to construct specialized interstellar emission models (IEMs) to characterize the

\footnotetext{
${ }^{1}$ Available at http://fermi.gsfc.nasa.gov/ssc/data/analysis

${ }^{2}$ Available at https://galprop.stanford.edu
} 

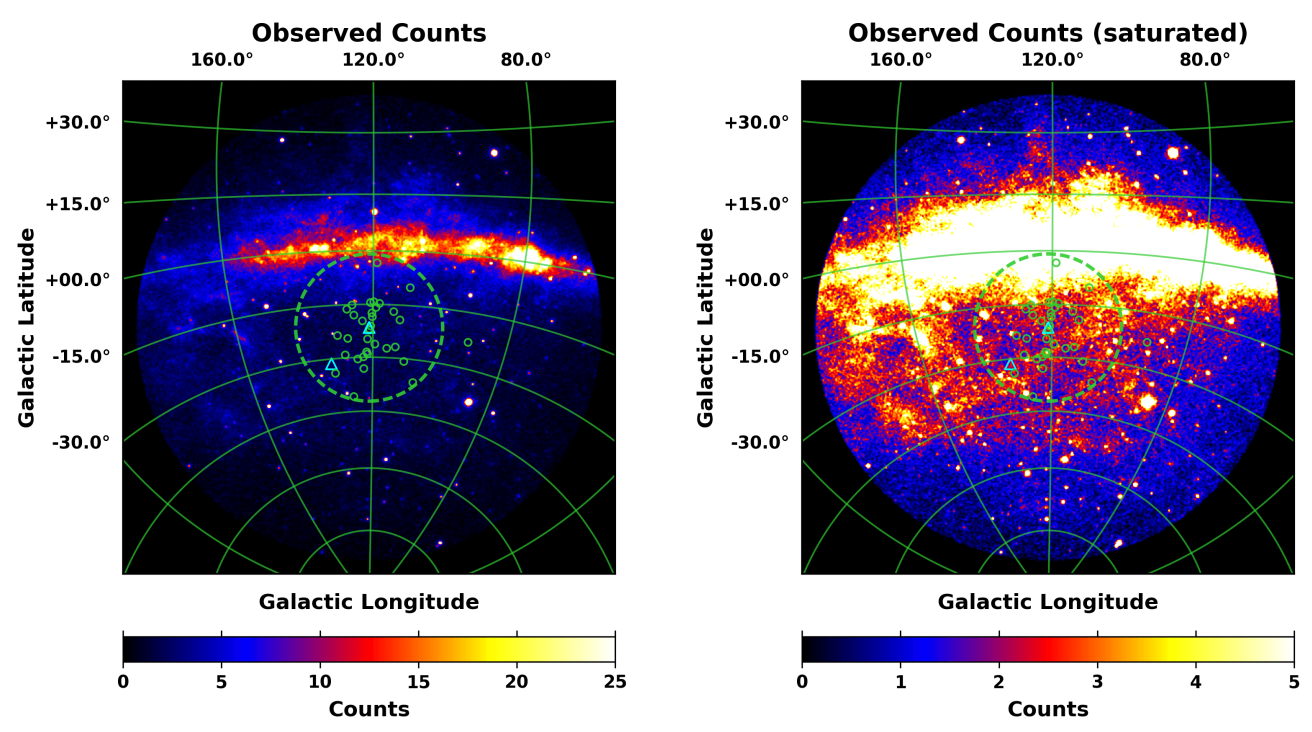

Figure 1: Observed counts (left) and saturated counts (right) for a $60^{\circ}$ radius centered at $\mathrm{M} 31$, and an energy range of $1-100 \mathrm{GeV}$. The green dashed circle $\left(21^{\circ}\right.$ in radius) corresponds to a $300 \mathrm{kpc}$ projected radius centered at $\mathrm{M} 31$, for an $\mathrm{M} 31-\mathrm{MW}$ distance of $785 \mathrm{kpc}$, i.e. the canonical virial radius of M31. Also shown is M31's population of dwarf galaxies. M31 and M33 are shown with cyan triangles, and the other dwarfs are shown with $1^{\circ}$ green circles, each centered at the optical center of the respective galaxy.

MW foreground emission, including a self-consistent determination of the isotropic component. These foreground models are physically motivated and are not subject to the same caveats ${ }^{3}$ for extended source analysis as the default IEM provided by the Fermi-LAT Collaboration for point source analysis [8].

The parameters of the GALPROP model are tuned to the measured local interstellar spectra (LIS) of CRs, including the latest AMS-02 measurements. We have adopted the best-fit parameters from the tuning procedure performed in $[9,10]$, where GALPROP and HelMod ${ }^{4}$ are implemented in an iterative manner, thereby accounting for solar modulation in a physically motivated way when fitting to the local CR measurements. The LIS are shown in Figure 2.

Figure 3 shows the total interstellar emission model, which consists of individual components for $\pi^{0}$-decay, inverse Compton (IC), and Bremsstrahlung. The components are defined in Galctocentric annuli. In total there are 8 annuli, but for FM31 only annulus 5 (the local annulus) and beyond contribute to the foreground emission. FM31 has a significant emission associated with $\mathrm{H} \mathrm{I}$ gas, but there is very little emission from $\mathrm{H}_{2}$ gas. A uniform spin temperature of $150 \mathrm{~K}$ is assumed for the baseline IEM. The foreground emission from $\mathrm{H}$ II and Bremsstrahlung are subdominant. Our model also accounts for the dark neutral medium (DNM). The anisotropic formalism is employed for the calculation of the IC component. In addition to the GALPROP IEM components, we also use a template approach to account for inaccuracies in the foreground model relating to the neutral gas along the line of sight. To model the point sources in the region, we employ the 3FGL as a starting point, and because of the larger statistics of our data set, we account for addi-

\footnotetext{
${ }^{3}$ For more information see https://fermi.gsfc.nasa.gov/ssc/data/analysis/LAT_caveats.html

${ }^{4}$ Available at http://www.helmod.org
} 

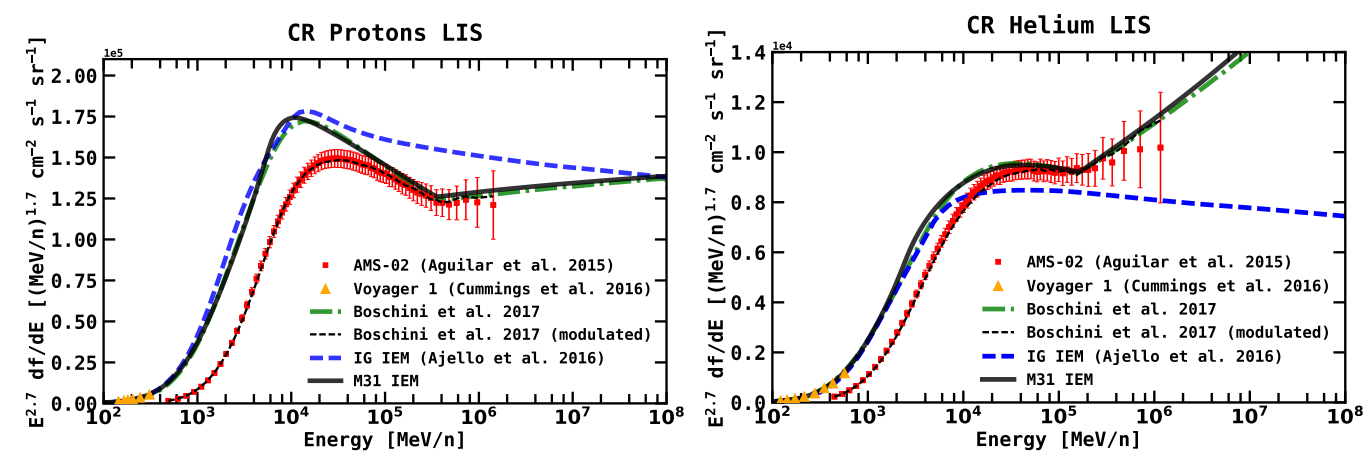

CR Electrons + Positrons LIS

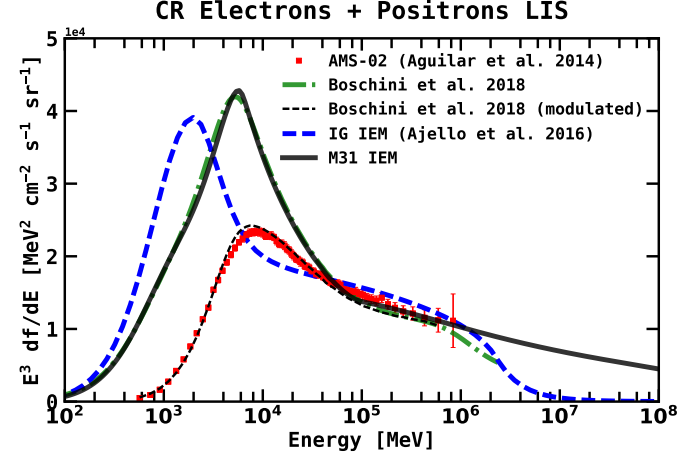

Figure 2: The local interstellar spectra (LIS) for CR protons, He, and all electrons $\left(e^{-}+e^{+}\right)$. The latest AMS-02 measurements from [11-13] are shown with red squares. The green dashed line shows the results from $[9,10]$, which employ GALPROP and HelMod together in an iterative manner to derive the LIS. We adopt their derived GALPROP CR parameters, and the LIS for our IEM (M31 IEM: solid black line) are roughly the same. The thin dotted black line shows the LIS modulated with HelMod $[9,10]$. Yellow triangles show the Voyager $1 p$ and $\mathrm{He}$ data in the local interstellar medium [14]. Voyager 1 electron data are below $100 \mathrm{MeV}$ and, therefore, are not shown. In addition we show the LIS for the ("Yusifov") IEM in [15], which we use as a reference model in our study of the systematics for the M31 field.

tional point sources self-consistently with the M31 IEM by implementing a point source finding procedure, which is based on a wavelet transform algorithm.

We calculate the isotropic component self-consistently with the M31 IEM. The main calculation is performed over the full sky in the following region: $|b| \geq 30^{\circ}, 45^{\circ} \leq l \leq 315^{\circ}$. To better determine the normalization of the isotropic component we use a tuning region (TR) directly below FM31, outside of the virial radius. The best-fit normalization is found to be $1.06 \pm 0.04$, and this remains fixed for all other fits with the M31 IEM. The fit in the TR yields a model that describes the data well across the entire region and at all energies. The best-fit normalizations of the IEM components in the TR are all in reasonable agreement with the GALPROP predictions. The spectra for the IEM components (as well as the isotropic component) and the remaining fractional count residuals for the fit in the TR are shown in the left panel of Figure 4.

\section{Results for the M31 Field}

The baseline fit in FM31 results in positive residual emission in the fractional count residuals between $\sim 3-20 \mathrm{GeV}$ at the level of $\sim 5 \%$, as seen in the right panel of Figure 4. A significant 


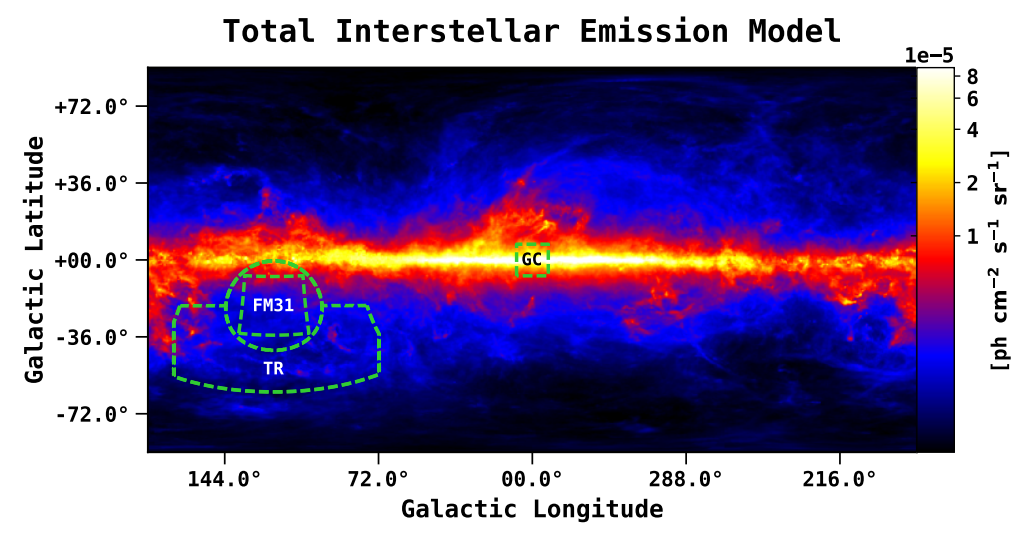

Figure 3: The total interstellar emission model (IEM) for the MW integrated in the energy range 1-100 $\mathrm{GeV}$. The color corresponds to the intensity, and is shown in logarithmic scale. The model has contributions from $\pi^{0}$-decay, (anisotropic) IC emission, and Bremsstrahlung. Overlaid is the region of interest (ROI) used in this analysis. From the observed counts (Figure 1) we cut an $84^{\circ} \times 84^{\circ} \mathrm{ROI}$, which is centered at M31. The green dashed circle is the $300 \mathrm{kpc}$ boundary corresponding to M31's canonical virial radius (of $\sim 21^{\circ}$ ), as also shown in Figure 1. We label the field within the virial radius as field M31 (FM31), and the region outside (and south of latitudes of $-21.57^{\circ}$ ) we label as the tuning region (TR). For reference we also show the Galactic center region (GC), which corresponds to a $15^{\circ} \times 15^{\circ}$ square centered at the GC.

fraction of the structured excess emission in FM31 is found to be spatially correlated with the H I column density and the foreground dust, including regions where the dust is relatively cold (see [5] for full details). This may be indicative of a spatially varying spin temperature. Correspondingly, the structured residual emission may be related to inaccuracies in the modeling of the DNM, which is determined as part of an all-sky procedure. We, therefore, refine the baseline IEM by constructing a template to account for potential mis-modeling of these components. The template is obtained by selecting the excess emission in FM31 that correlates with $\mathrm{H}$ I tracers. We refer to this as the arc template. This procedure accounts for any un-modeled H I (or other Galactic gas), as well as any mis-modeling in its line of sight distance, spin temperature, and spectral index variations. We find that the specialized IEMs for the analysis of FM31, both the baseline model and the baseline model with the arc template, yield an extended excess at the level of $\sim 5 \%$ in the $\sim 3-20 \mathrm{GeV}$ energy range. We show that the excess is also present with similar characteristics when alternative IEMs are employed, and when systematic variations of the spectrum of point sources are considered.

To determine whether the excess presents a spherically symmetric gradient about the center of M31, which would lend support to the hypothesis that it originates from there (at least partially), we perform a further fit in FM31 by including three symmetric uniform templates centered at M31. This also allows us to quantify the spectrum and gradient of the positive residual emission. The templates are fit concurrently with the other components of the baseline IEM, including the arc template. The inner disk (inner galaxy) has a radial extension of $0.4^{\circ}(5.5 \mathrm{kpc}$ projected radius). The intermediate ring (spherical halo) has a radial extension from $0.4^{\circ}<r \leq 8.5^{\circ}$ (117 kpc projected radius). The outer ring (far outer halo) covers the remaining extent of FM31, corresponding to a total projected radius of $\sim 200 \mathrm{kpc}$, and likewise it begins to approach the MW plane towards the top of the field. We find that all templates are significantly detected (with a significance of 

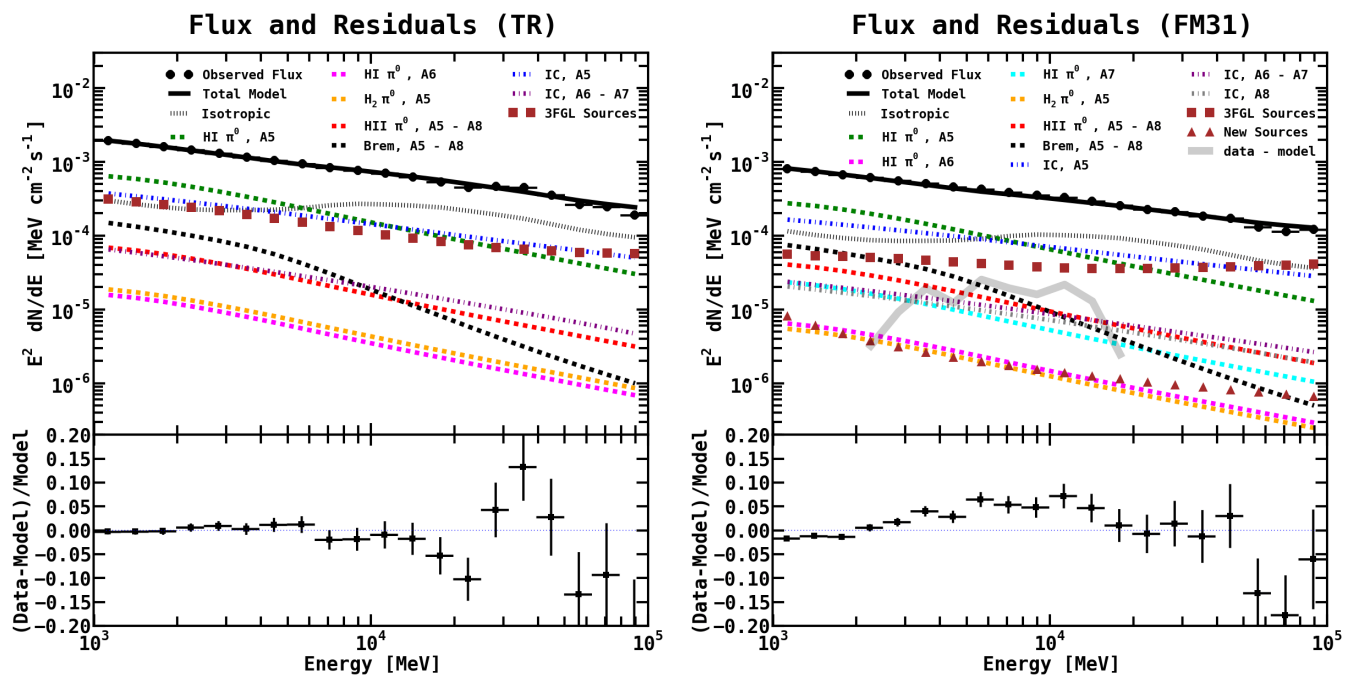

Figure 4: Flux (upper panel) and fractional count residuals (lower panel) for the fit in the TR (left) and the baseline fit in FM31 (right).

$\geq 5 \sigma$ ). Furthermore, the M31-related components are able to flatten the positive residual emission in the fractional count residuals.

The spectrum and intensity for the inner galaxy are consistent with previously published results. The spherical halo and far outer halo have intensities that are much dimmer than the inner galaxy, and present a mild intensity gradient, tapering off with distance from the center of M31, as shown in the left panel of Figure 5. Their spectra are significantly different from all the other extended components in FM31. They peak between $\sim 5-10 \mathrm{GeV}$, and drop off below and above these energies more steeply than all other contributions. We find it difficult to reconcile these spectra with the possibility that the excess emission originates solely within the MW, further setting it apart from known Galactic sources. Beyond these general features, the spectra for the two outer annuli differ from each other with the far outer halo presenting a harder spectrum at low energies. The best-fit spectra for the FM31 spherical halo and far outer halo components are shown in the right panel of Figure 5. We compare the spectral shapes to the systematic band of the Galactic center excess (for an arbitrary normalization) from [15], and find that they are qualitatively consistent, as can be seen in the Figure.

Our results show that if the excess emission originates (at least partially) from the M31 system, its extension may reach a distance upwards of $\sim 120-200 \mathrm{kpc}$ from the center of M31. This is consistent with the expectation for a DM signal, as the virial radius for the DM halo extends at least this far. To test this interpretation, in the left panel of Figure 5 we compare the radial profile of the observed excess with predictions for a DM signal, including both the M31 halo and the MW halo along the line of sight, with a spectrum and annihilation cross-section consistent with a DM interpretation of the Fermi-LAT GC excess $[15,17]$. We consider different assumptions for the amount of DM substructure in M31 (and the MW), and we find that if a cold DM scenario is assumed that includes a large boost factor due to substructures (although within theoretical expectations), the observed excess emission is consistent with this interpretation. Granted, however, the exact parti- 

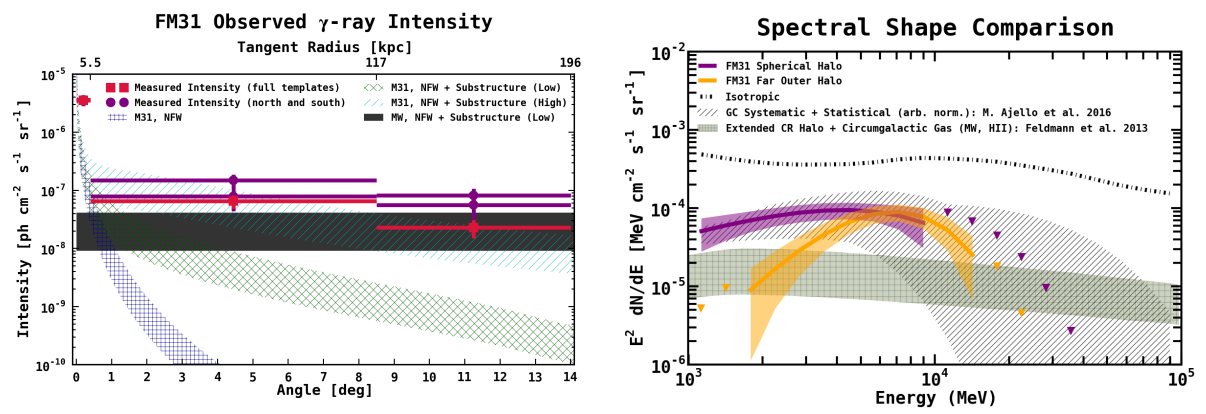

Figure 5: Left: Radial intensity profile for the M31-related components. For reference, we compare the radial profile to expectations for DM annihilation in the line of sight, including the contribution from the MW halo. Right: Spectral shape comparison to the Galactic center excess (for an arbitrary normalization), as observed in [15]. Also shown is a prediction for CRs interacting with the ionized gas of the circumgalactic medium from [16].

tioning of individual contributions to the signal remains unclear, i.e. primary emission from M31's DM halo, secondary emission in M31, emission from the local DM filament between M31 and the MW, and emission from the MW's DM halo along the line of sight. This is an intriguing finding, however, its implications are far reaching, and better understanding the MW foreground, as well as complementarity with other DM targets, is crucial before drawing any stronger conclusions.

\section{Summary}

We present the first search for extended emission from M31 in $\gamma$-rays out to a distance of $\sim 200$ $\mathrm{kpc}$ from its center (see [5] for full analysis). We find evidence for an extended excess that appears to be distinct from the conventional MW foreground, having a total radial extension upwards of 120-200 kpc from the center of M31. We discuss plausible interpretations for the excess emission, but emphasize that uncertainties in the MW foreground, and in particular modeling of the $\mathrm{H} \mathrm{I}$ related components, have not been fully explored and may impact the results. We find that a DM interpretation provides a good description of the observed emission and is consistent with the GC excess DM interpretation. However, better understanding of the systematics, and complementarity with other DM searches, is critical to settle the issue.

\section{Acknowledgements}

The work of C.K. and S.M. is supported in part by Department of Energy grant DESC0014431. S.C. is supported by National Science Foundation Grant PHY-1620638 and a McCue Fellowship. I.M. acknowledges partial support from NASA grant NNX17AB48G.

The Fermi-LAT Collaboration acknowledges support for LAT development, operation and data analysis from NASA and DOE (United States), CEA/Irfu and IN2P3/CNRS (France), ASI and INFN (Italy), MEXT, KEK, and JAXA (Japan), and the K.A. Wallenberg Foundation, the Swedish Research Council and the National Space Board (Sweden). Science analysis support in the operations phase from INAF (Italy) and CNES (France) is also gratefully acknowledged. This work performed in part under DOE Contract DE-AC02-76SF00515. 


\section{References}

[1] FERMI-LAT collaboration, Fermi Large Area Telescope observations of Local Group galaxies: Detection of M31 and search for M33, A\&A 523 (2010) L2 [1012.1952].

[2] H. Ögelman, N. Aksaker, S. Anılan, H. Dereli, N. Emrahoğlu and I. Yegingil, Discovery of gamma-ray emission from M31 via Fermi-LAT, AIP Conference Proceedings 1379 (2011) 82.

[3] M. S. Pshirkov, V. V. Vasiliev and K. A. Postnov, Evidence of Fermi bubbles around M31, MNRAS 459 (2016) L76 [1603.07245].

[4] FERMI-LAT collaboration, Observations of M31 and M33 with the Fermi Large Area Telescope: A Galactic Center Excess in Andromeda?, ApJ 836 (2017) 208 [1702 . 08602 ].

[5] C. Karwin, S. Murgia, S. Campbell and I. Moskalenko, Fermi-LAT Observations of Gamma-Ray Emission Towards the Outer Halo of M31, 1903.10533.

[6] I. V. Moskalenko and A. W. Strong, Production and propagation of cosmic ray positrons and electrons, ApJ 493 (1998) 694 [astro-ph/9710124].

[7] A. W. Strong and I. V. Moskalenko, Propagation of cosmic-ray nucleons in the galaxy, ApJ 509 (1998) 212 [astro-ph/9807150].

[8] FERMI-LAT collaboration, Development of the Model of Galactic Interstellar Emission for Standard Point-Source Analysis of Fermi Large Area Telescope Data, ApJS 223 (2016) 26 [1602 . 07246 ].

[9] M. J. Boschini, S. Della Torre, M. Gervasi, D. Grandi, G. Jóhannesson, M. Kachelriess et al., Solution of Heliospheric Propagation: Unveiling the Local Interstellar Spectra of Cosmic-ray Species, ApJ 840 (2017) 115 [1704.06337].

[10] M. J. Boschini, S. Della Torre, M. Gervasi, D. Grandi, G. Jóhannesson, G. La Vacca et al., HelMod in the Works: From Direct Observations to the Local Interstellar Spectrum of Cosmic-Ray Electrons, ApJ 854 (2018) 94 [1801.04059].

[11] AMS COLlaboration collaboration, Precision measurement of the $\left(e^{+}+e^{-}\right)$flux in primary cosmic rays from $0.5 \mathrm{gev}$ to $1 \mathrm{tev}$ with the alpha magnetic spectrometer on the international space station, PhRvL 113 (2014) 221102.

[12] AMS COllaboration collaboration, Precision measurement of the proton flux in primary cosmic rays from rigidity $1 \mathrm{gv}$ to $1.8 \mathrm{tv}$ with the alpha magnetic spectrometer on the international space station, PhRvL 114 (2015) 171103.

[13] AMS COllaboration collaboration, Precision measurement of the helium flux in primary cosmic rays of rigidities $1.9 \mathrm{gv}$ to $3 \mathrm{tv}$ with the alpha magnetic spectrometer on the international space station, PhRvL 115 (2015) 211101.

[14] A. C. Cummings, E. C. Stone, B. C. Heikkila, N. Lal, W. R. Webber, G. Jóhannesson et al., Galactic Cosmic Rays in the Local Interstellar Medium: Voyager 1 Observations and Model Results, ApJ 831 (2016) 18.

[15] FERMI-LAT collaboration, Fermi-LAT Observations of High-Energy $\gamma$-Ray Emission Toward the Galactic Center, ApJ 819 (2016) 44 [1511.02938].

[16] R. Feldmann, D. Hooper and N. Y. Gnedin, Circum-Galactic Gas and the Isotropic Gamma Ray Background, ApJ 763 (2013) 21 [1205.0249].

[17] D. Hooper and L. Goodenough, Dark Matter Annihilation in The Galactic Center As Seen by the Fermi Gamma Ray Space Telescope, PhL B697 (2011) 412 [1010 . 2752]. 